\title{
Conjugate mirror by a panda ring circuit
}

\author{
Preecha P. Yupapin ${ }^{1, *}$, Napatsakorn Sarapat ${ }^{2}$ \\ ${ }^{1}$ Advanced Studies Center, Department of Physics, Faculty of Science, King Mongkut's Institute of Technology Ladkrabang (KMITL), \\ Bangkok 10520, Thailand \\ ${ }^{2}$ Faculty of Science and Technology, Tepsatri Rajabhat University, Lopburi 15000, Thailand
}

\section{Email address:}

kypreech@kmitl.ac.th (P. P. Yupapin)

\section{To cite this article:}

Preecha P. Yupapin, Napatsakorn Sarapat. Conjugate Mirror by a Panda Ring Circuit, Science Innovation. Vol. 1, No. 1, 2013, pp. 1-4. doi: $10.11648 /$ j.si.20130101.11

\begin{abstract}
This paper presents the use of a nonlinear microring resonator device known as a modified add-drop filter for small scale conjugate mirror, which is a basic device for 3D image construction and reconstruction system. It can also be useful for real time 3D image display applications. By using the modified add-drop filter, the 3D image can be constructed and reconstructed within the microring circuit. In this circuit, the object and reference beams can be formed by the reflected signals from through and drop ports of the add-drop filter respectively, where the conjugate mirror concept is obtained by the nonlinear coupling effects via the two nonlinear side rings, in which the four-wave mixing of those signals can be introduced. Finally, the interference of those two beams can be constructed and seen by the whispering gallery mode at the center ring, while the interference signals can be seen by the add port output. The WGMs of waves are simulated and discussed, where the $3 \mathrm{D}$ image construction and reconstruction can be seen by using the microring circuit as the conjugate mirror, in which the use of large volume display using a new type of conjugate mirror can be realized.
\end{abstract}

Keywords: Conjugate Mirror, 3D Display, 3D Image, Holography, Wgms

\section{Introduction}

The 3D image technology known as "holography" has become the promising technology today, which has been continuously developed for many years, especially, when it is applied to use in recent technologies such as television, movies and mobile phone. There is a revolutionary concept telephone, in which part of the telephone screen using holographic technique for applications is required. Practically, the important device that can be used to construct the 3D images is the optical phase conjugation components [1, 2], which can be functioned by the four-wave mixing technique, though it is also possible to use the process in several ways $[3,4]$. An important device that uses to produce the phase conjugation effect is known as a phase conjugate mirror (PCM), which is the key device to work in holographic applications. Till now, however, we still search for the new techniques that can show the advantage over the past components. Recently, Yupapin [5] have proposed the very interesting technique that uses WGMs in a PANDA ring waveguide for many applications [6, 7].

In this present work we design a new structure of device which consists of a nonlinear microring resonator (see Ref. [8]) and two phase conjugate mirrors as shown in Fig. 1, which will be used to construct and reconstruct the 3D images for display application, in which the object and reference beams can be configured by the through and drop ports of the modified add-drop filter, where the conjugate mirror behavior can be obtained by the nonlinear coupling effects from the two nonlinear side rings as shown in Figure 2. Finally, the interference signals of those two beams can be constructed and seen by the whispering gallery mode at the center ring, which is the real time images, while the reference interference signals can be detected by the add port output, which is required the reconstruction process similar to the old holographic technique.

The large volume structure is also available using thin film device which is useful for flat panel $3 \mathrm{D}$ image applications. More applications such as flat panel holographic display, holographic sensor, infrared holography etc. can be produces. The device scale is within 10-50 microns which can be fabricated and implemented, the center ring device material can be a linear or nonlinear material, while the side ring material must be the nonlinear material that gives the nonlinear effects coupling to the center ring, which is allowed the occurrence of four-wave mixing, i.e. conjugate mirror requirement. In this work, In $\boldsymbol{G a} \boldsymbol{A s} \boldsymbol{P} / \mathbf{I n} \boldsymbol{P}$ is proposed as the required nonlinear material. Finally, the device di- 
mension can be currently available in the fabrication industry. The theoretical review and some results of 3D image are given in the next following sections.

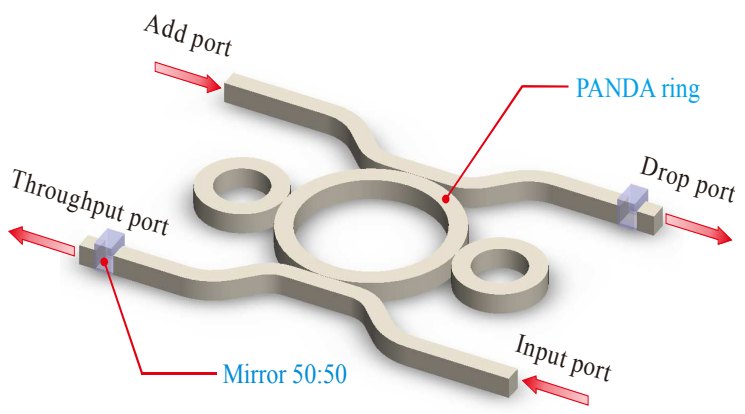

Figure 1. A schematic structure of conjugate mirror system

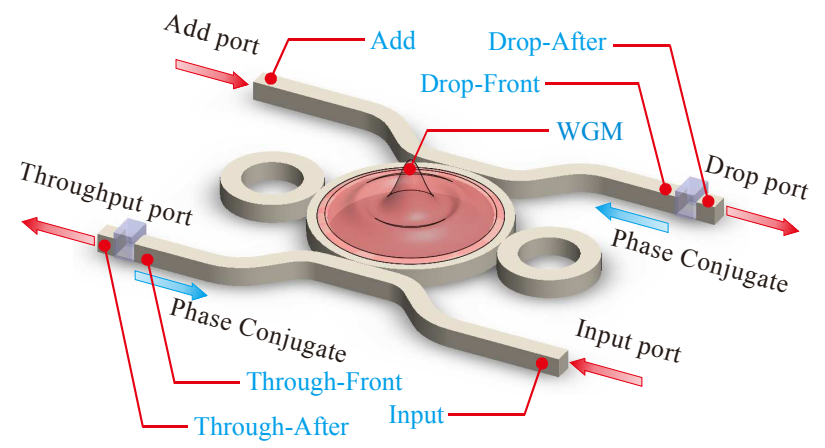

Figure 2. Shows the probe signal positions of the conjugate mirror

\section{Whispering Gallery Modes (WGMs)}

A PANDA ring resonator is as shown in Figures 1 and 2, where the electromagnetic field in the form of WGM can be described by time-dependent Maxwell's equations and given by reference [9], where more details and references can be found in references $[10,11]$. In general, the whispering gallery mode result is obtained by using the Optiwave program as shown in Figure 3(a), where the signals were changed to obtain the WGMs in the conjugate mirror mode as shown in Figure 3(b), i.e. the reflected signals are now taken in the simulation. The ring material is In-

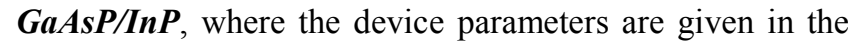
figure caption, which is the micrometer scale. In this article, the full-wave finite-difference time-domain (FDTD) method is employed to solve Maxwell's equations. The FDTD modeling gives a useful design role, which is the combination of the propagation, scattering, diffraction, reflections, and polarization effects. It also handles well the material anisotropy, dispersion and nonlinearities without any pre-assumption of field behavior as the slowly varying amplitude approximation [9]. When a two dimensional (2-D) problem is included, the photonic device is laid out in the $\mathrm{X}-\mathrm{Z}$ plane, the propagation is along the $\mathrm{Y}$-axis, and the $\mathrm{Z}$ direction is assumed to be infinite and there is no variation in the Z-direction. The parameters are obtained by using the related practical material parameters of InGaAsP/InP. Here, the waveguide core $n=3.14$ is bordered on each side by air $\mathrm{n}=1$. The parameters for add-drop optical multiplexer and both nanorings on the left and right hand sides of the PANDA ring are set at $\mathrm{R}_{1}=\mathrm{Rr}=0.775 \mu \mathrm{m} \mu \mathrm{m}$ and radius of the center ring is $\mathrm{R}_{\mathrm{ad}}=1.56 \mu \mathrm{m}$. The coupling coefficient ratios are $\kappa_{1}=\kappa_{4}=0.5, \kappa_{2}=\kappa_{3}=0.5$, effective core area of the waveguides is $A_{\text {eff }}=0.25 \mu \mathrm{m}^{2}$, and waveguide loss coefficient is $\alpha=0.1 \mathrm{~dB} / \mathrm{mm}$.

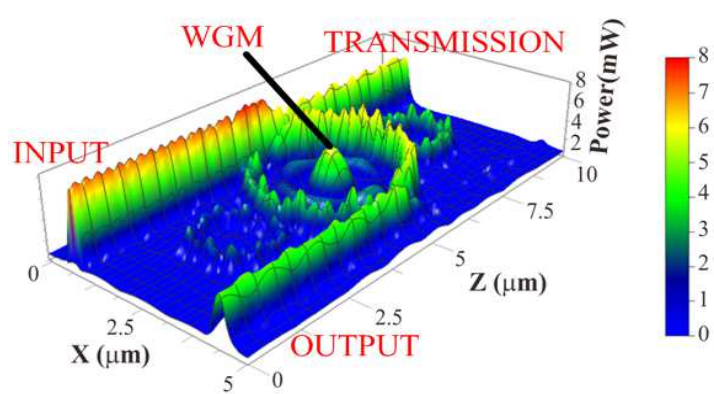

(a)

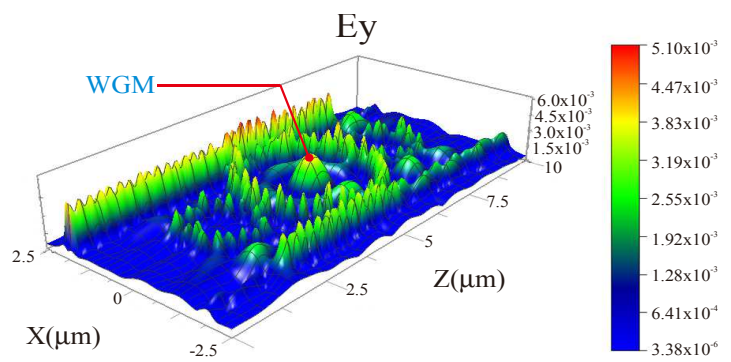

(b)

Figure 3. (a) result of whispering gallery mode of light within a PANDA ring waveguide InGaAsP/InP, $R_{1}=R_{2}=0.775 \mu \mathrm{m}, R_{a d}=1.565, A_{\text {eff }}=0.3$ $\mu \mathrm{m}^{2}, n_{\text {eff }}=3.14, n_{2}=1.3 \times 10^{-13} \mathrm{~cm}^{2} / W, \kappa_{1}=\kappa_{2}=\kappa_{3}=\kappa_{4}=0.5, \gamma=0.01, \lambda_{0}$ $=1,550 \mathrm{~nm}$, (b) shows the WGMs by conjugate mirror system.

Figure 4 shows the nice display of the conjugate mirror signals within a proposed device, in which the phase conjugate signals are as shown in Figures 5-9. In principle, the four-wave mixing is introduce into the center ring by the nonlinear coupling from the two side rings, the interference signals are formed by the reflected signals from the Through and Drop ports, where finally the object and reference beams within the device can be performed. However, in this case, the real time 3D display can be formed simultaneously with the constructed signals via the Add port, which will be available for large required applications

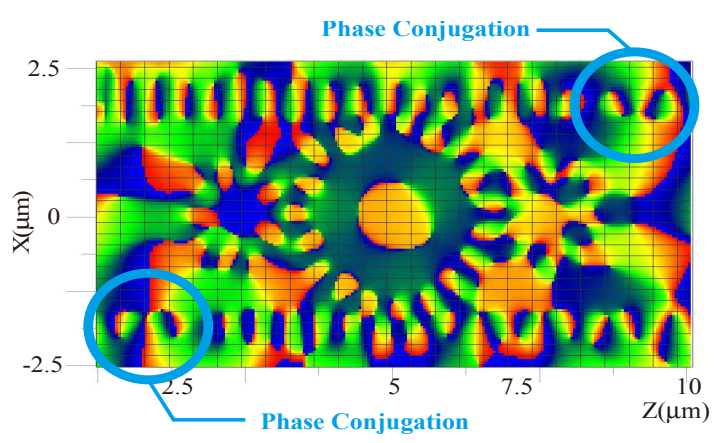

Figure 4. Shows signals in the conjugate mirror system. 


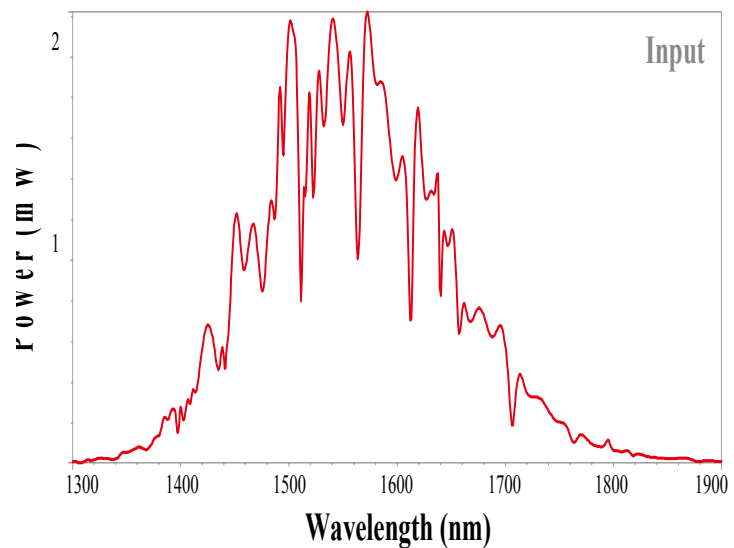

Figure 5. Result of Input signal in the wavelength domain.

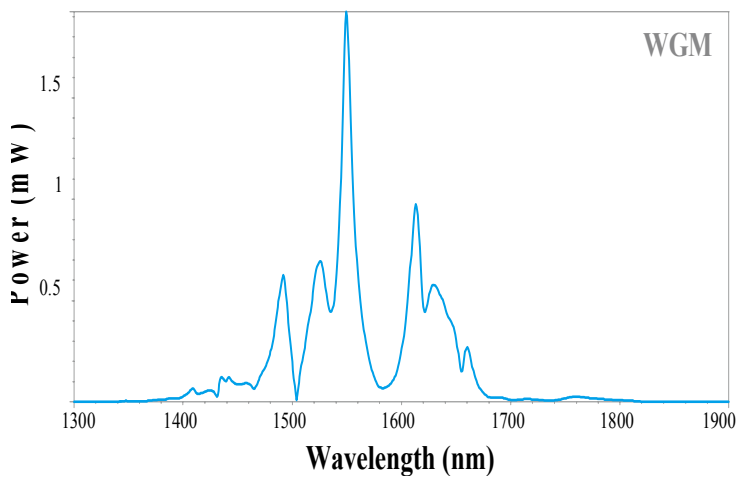

Figure 6. Result of WGM signal in the wavelength domain.
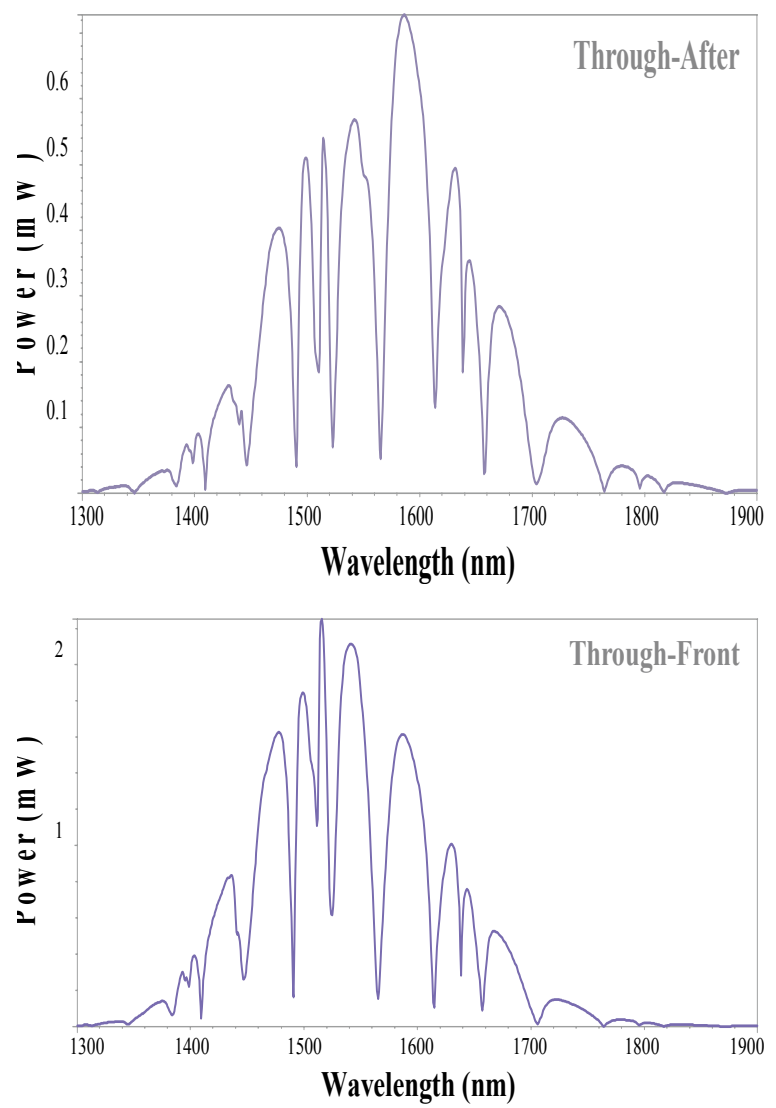

Figure 7. Result of Through port signal in the wavelength domain.
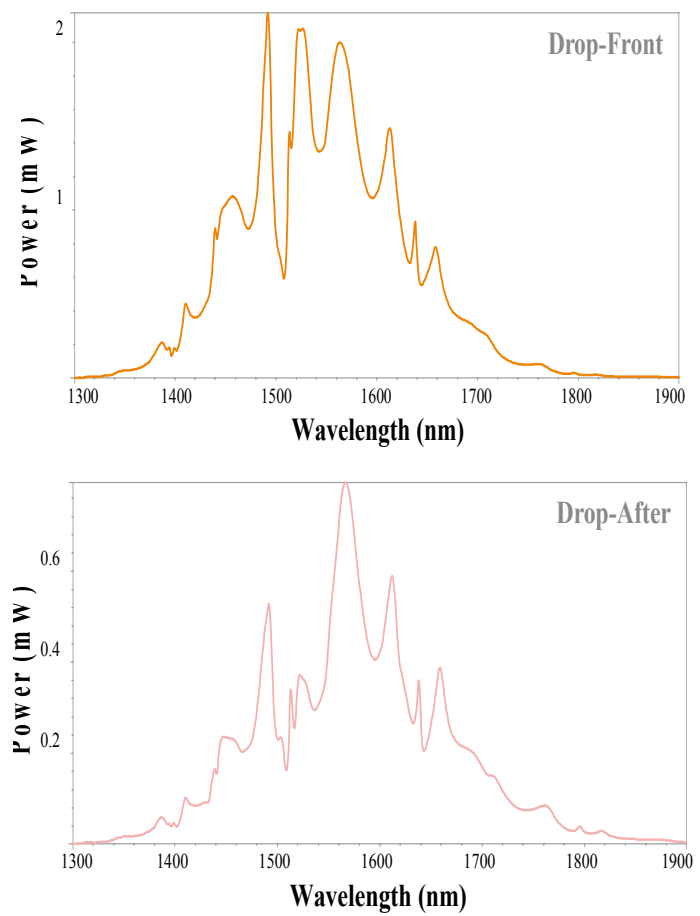

Figure 8. Result of the Drop port signal in the wavelength domain.

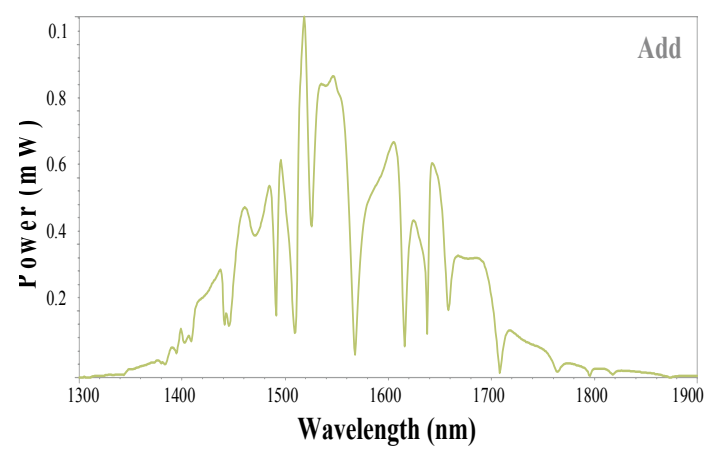

Figure 9. Result of the Add port signal in the wavelength domain.

From Figure 4, the center signal will display the real time $3 \mathrm{D}$ images, which is the same as the Add port output signals. But in this case we can see the real time images by naked eyes, while the constructed images from the Add port can be seen after computerized signals. Moreover, this device dimension and material can be processed and available in the industry, which can be the large demand of the world in the near future.

\section{Conclusion}

We have shown the very interesting device that can be potentially used for new type of holographic display applications. By using the nonlinear effects within a PANDA ring, the new type conjugate mirror can be formed and realized. The four waves mixing of waves can be introduced and the holographic image can be performed within the circuit, the 3D image signals can also be seen simultaneously by the Add port constructed images. In application, the large area (volume) of 3D image device can be constructed by making a thin film flat panel, which can be used to serve the large 
demand of applications. The use of the proposed device is also available for sensor applications, which will be our continuing work.

\section{Acknowledgment}

We would like to thank the King Mongkut's Institute of Technology Ladkrabang (KMITL), Thailand for providing the research facilities.

\section{References}

[1] V. Shkunov and B. Zel'dovich, "Phase conjugation", Scientific American, 253 (1985), 54-59.

[2] D. M. Pepper, "Applications of optical phase conjugation", Scientific American, 254 (1986), 74-83.

[3] D. Hounam, A SAR conjugate mirror, IEEE Geoscience and Remote Sensing Letters, 3(3), (2006), 373-376.

[4] E.J. Bochove, Theory of a variable aperture phase conjugate mirror with application to an optical cavity, J. Applied Physics, 59(10), (1986), 3360-3362.

[5] P.P. Yupapin, "Nonlinear coupling effects of waves in a
PANDA ring," Discovery Science, 1(1), 2013.

[6] J.C. Knight, N. Dubreuil, V. Sundoghdar, J. Hare, V. Lefevre-Seguin, J.M. Raimond and S. Haroche, "Characterizing whispering-gallery modes in microspheres by direct observation of the optical standing-wave pattern in the near field", Optics Letters, 21(10)(1996)698-670.

[7] N. Thammawongsa, S. Tunsiri, M.A. Jalil, J. Ali and P.P. Yupapin, "Storing and harvesting atoms/molecules On-Chip: Challenges and applications", J. Biosensors \& Bioelectronics, 3(5)(2012) e114-115.

[8] N. Sarpat, W. Khunnam, S. Chiangag, N. Thammawongsa, M.A. Jalil, J. Ali and P.P. Yupapin, Fast, Slow, Stopping and Storing Light Simultaneously using a PANDA Ring On-Chip," Asia Pacific Physics Newsletter, 2(1) 2013.

[9] K.S. Yee, "Numerical solution of initial boundary value problems involving Maxwell's equations in isotropic media", IEEE Transaction on Antennas Propagation, 14, (1996)302-307 (1966).

[10] E. Waks, V. Jelena, "Coupled mode theory for photonic crystal cavity-waveguide interaction", Optics Express, 13(13)(2005) 5064-5073.

[11] N. Thammawongsa, N. Moonfangklang, S. Mitatha, P.P. Yupapin, "Novel nano-antenna system design using photonics spin in a panda ring resonator", PIER L, 31(2012) 75-87. 